\title{
Giant intrapelvic malignant peripheral nerve sheath tumor mimicking disc herniation: A case report
}

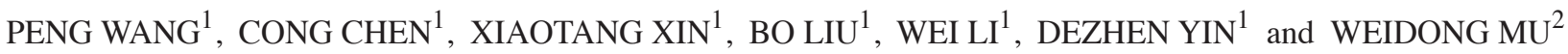 \\ ${ }^{1}$ Department of Orthopaedics, Weihai Municipal Hospital, Weihai, Shandong 264200; ${ }^{2}$ Department of Traumatic \\ Orthopaedics, Shandong Provincial Hospital Affiliated to Shandong University, Jinan, Shandong 250012, P.R. China
}

Received March 30, 2016; Accepted September 15, 2016

DOI: $10.3892 / \operatorname{mco} .2016 .1030$

\begin{abstract}
Giant intrapelvic malignant peripheral nerve sheath tumors arising in the sciatic nerve in the pelvic cavity are a rare occurrence and their symptomatology is usually misdiagnosed as intervertebral disc herniation. We herein report the case of a 46-year old woman presenting with pain, hypesthesia and weakness of the left lower extremity due to a giant intrapelvic malignant peripheral nerve sheath tumor of the sciatic nerve. Prior to being referred to our institution, the patient was misdiagnosed as a case of sciatica due to a lumbar disc herniation and underwent an operation unsuccessfully, as there was little symptomatic improvement 2 months after the surgery. A magnetic resonance imaging examination of the pelvic cavity revealed a tumor of the sciatic nerve. The mass was resected via the posterior approach and histopathological examination confirmed the diagnosis of malignant peripheral nerve sheath tumor. Intrapelvic malignant peripheral nerve sheath tumors are an uncommon cause of sciatica and are commonly misdiagnosed as lumbar intervertebral disc herniation. Accurate diagnosis and complete surgical excision prior to metastasis are crucial for effective management of this condition.
\end{abstract}

\section{Introduction}

The most common cause of sciatica is intervertebral disc herniation; thus, obscure causes are rarely considered in the differential diagnosis. Malignant peripheral nerve sheath tumor (MPNST) arising from the sciatic nerve is a relatively rare malignant soft tissue tumor (1). This tumor may produce severe radicular pain and sensorimotor deficits, mimicking a disc herniation. Due to the rare incidence of this disease, reports on intrapelvic MPNSTs are scarce and there has been no systemic study of intrapelvic MPNSTs to date $(2,3)$. In this

Correspondence to: Dr Weidong Mu, Department of Traumatic Orthopaedics, Shandong Provincial Hospital Affiliated to Shandong University, 324 Jing Wu Road, Jinan, Shandong 250012, P.R. China E-mail: sdphweidongmu@126.com

Key words: malignant peripheral nerve sheath tumor, sciatica, lumbar intervertebral disc herniation study, we reported a misdiagnosed case of giant intrapelvic MPNST of the sciatic nerve mimicking disc herniation. Our aim was raise awareness that intrapelvic MPNST is an unusual cause of sciatica, which is usually misdiagnosed as disc herniation. The diagnosis, treatment and prognosis of MPNSTs were also discussed.

\section{Case report}

A 46-year old woman was admitted to another hospital with a 3-month history of pain that radiated from the posterior aspect of the left thigh to the lateral aspect of the leg and left foot, and was associated with numbness of the left lower limb. The patient had undergone a lumbar magnetic resonance imaging (MRI) examination, which revealed a mild L4/5 intervertebral disk herniation (Fig. 1A and B). A clinical diagnosis of intervertebral disk herniation was made, and L4/5 discectomy was performed (Fig. 1C and D). However, there was little symptomatic improvement after 2 months. The patient was subsequently referred to our institution.

On admission, the patient's neurological examination revealed grade $3 / 5$ weakness of the quadriceps femoris, grade $2 / 5$ weakness of the triceps surae and grade $0 / 5$ weakness of the extensor hallucis longus and extensor digitorum longus, a decreased sensation to light touch, decreased patellar reflex and Achilles reflex on the left, and a positive straight leg raise test. The patient underwent further examination by MRI and magnetic resonance angiogram (MRA). MRI and MRA of the pelvis revealed a mass sized $\sim 7-8 \mathrm{~cm}$ within the pelvis involving the internal iliac artery (Fig. 2A-C). A soft, smooth fixed mass in the left side of the pelvis was identified.

Gynecological and urological consultations were obtained. A decision was made to excise the tumor under general anesthesia. The intrapelvic tumor was exposed through the posterior approach (Fig. 2D). An incisional biopsy with intraoperative frozen section examination was performed from the extrapelvic part of the mass, and the result indicated a malignant tumor. After being exposed from the greater sciatic notch, a dumbbell mass was identified surrounding the sciatic nerve. The tumor tightly enclosed the sciatic nerve and it was difficult to separate the tumor from the nerve. Wide resection of the tumor and the invaded part of the sciatic nerve was performed from the proximal to the distal end (Fig. 2E). The final diagnosis of MPNST was confirmed by histopathological 

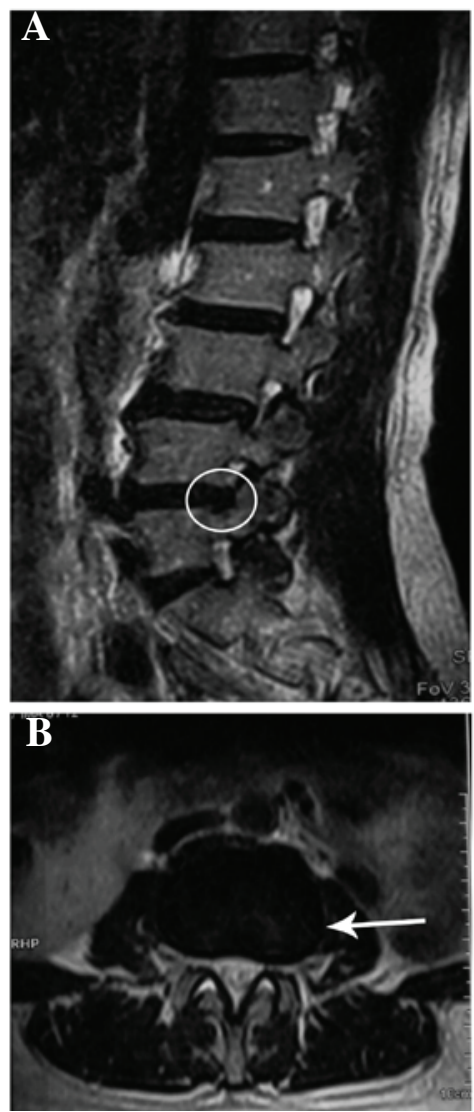
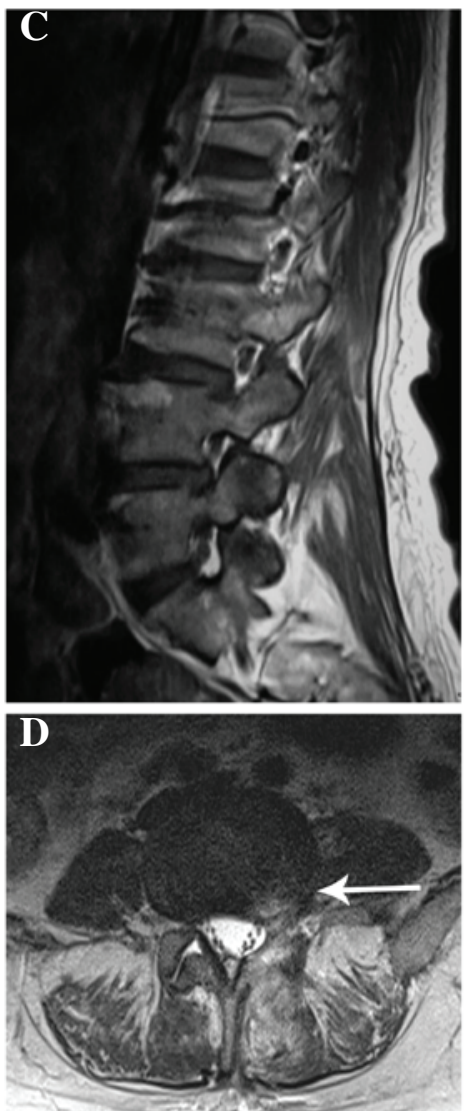

Figure 1. (A) T2-weighted sagittal view prior to L4/5 discectomy showing a left disc herniation at the L4-5 level with compression of the nerve root (circle). (B) T2-weighted axial view prior to L4/5 discectomy showing left lateral disc herniation at the L4-5 level (arrow). (C) T2-weighted sagittal view following L4/5 discectomy. (D) T2-weighted axial view showing high T2 signal of the surgical scar (arrow).

examination (Fig. 2F). The patient experienced loss of sensory and motor function below the left knee level after the operation. She refused further adjunctive radiotherapy and succumbed to pulmonary metastases 3 years postoperatively. Signed consent was obtained from the patient's family regarding the publication of the case details.

\section{Discussion}

MPNST is a rare soft tissue sarcoma, often originating from Schwann cells. While accounting for 3-10\% of all soft tissue sarcomas (4), the incidence of MPNST in the general population is $0.001 \%$ (5). The most important risk factor for MPNST development is the presence of neurofibromatosis type 1 (NF1), and $30 \%$ of MPNST cases occur in patients with a history of NF1 (6). In addition, MPNST may also develop as a result of therapeutic irradiation in populations that have previously undergone radiotherapy for malignant disease (7).

MPNSTs may occur anywhere along the peripheral nerves in the deep tissues. The significance of intrapelvic MPNST lies with its diagnostic difficulties in clinical practice. Pelvic tumors grow slowly and cannot be palpated along the nerve course. They are generally difficult to diagnose until they give rise to symptoms. The most common clinical presentation of intrapelvic MPNST consists of radicular and distal pain, paraesthesia and motor deficiencies.

Since the symptoms are similar to sciatica due to a herniated disk, while imaging of the spine may reveal discopathy, intrapelvic MPNST of the sciatic nerve is easily misdiagnosed $(8,9)$. A misinterpretation of the findings may result in incorrect treatment by discectomy, as reported in our patient. However, when there is no improvement of the symptoms following discectomy, the original diagnosis must be questioned (10). Ultrasonography, pelvic CT scan or MRI may be used to demonstrate the tumor along with the nerve. MRI is the most useful diagnostic tool, which allows assessment of the tumor's associations with adjacent structures. Information on the enhancing quality of the tumor, combined with its appearance on T1- and T2-weighted images, may offer some benefit in terms of differentiating benign peripheral nerve sheath tumors (BPNSTs) from MPNSTs. In the majority of the cases, central low signal intensity with a peripheral ring of high signal intensity on T2-weighted images are characteristic signs of a benign lesion (11), whereas a heterogeneous lesion due to necrosis and hemorrhage and patchy contrast enhancement may suggest malignancy (12). Although MRI may offer useful preoperative information, definitive diagnosis relies on pathology. Open biopsy is more accurate compared with percutaneous biopsy. To reduce the risk of contamination, the biopsy technique should be carefully considered, including proper location of the biopsy incision and meticulous hemostasis. The S-100 protein and Ki-67 index are widely used as immunohistochemical markers for MPNST.

Once the diagnosis of intrapelvic MPNST is suspected, surgical excision is the mainstay of treatment. The aim of surgery is complete removal of the lesion with tumor-free 

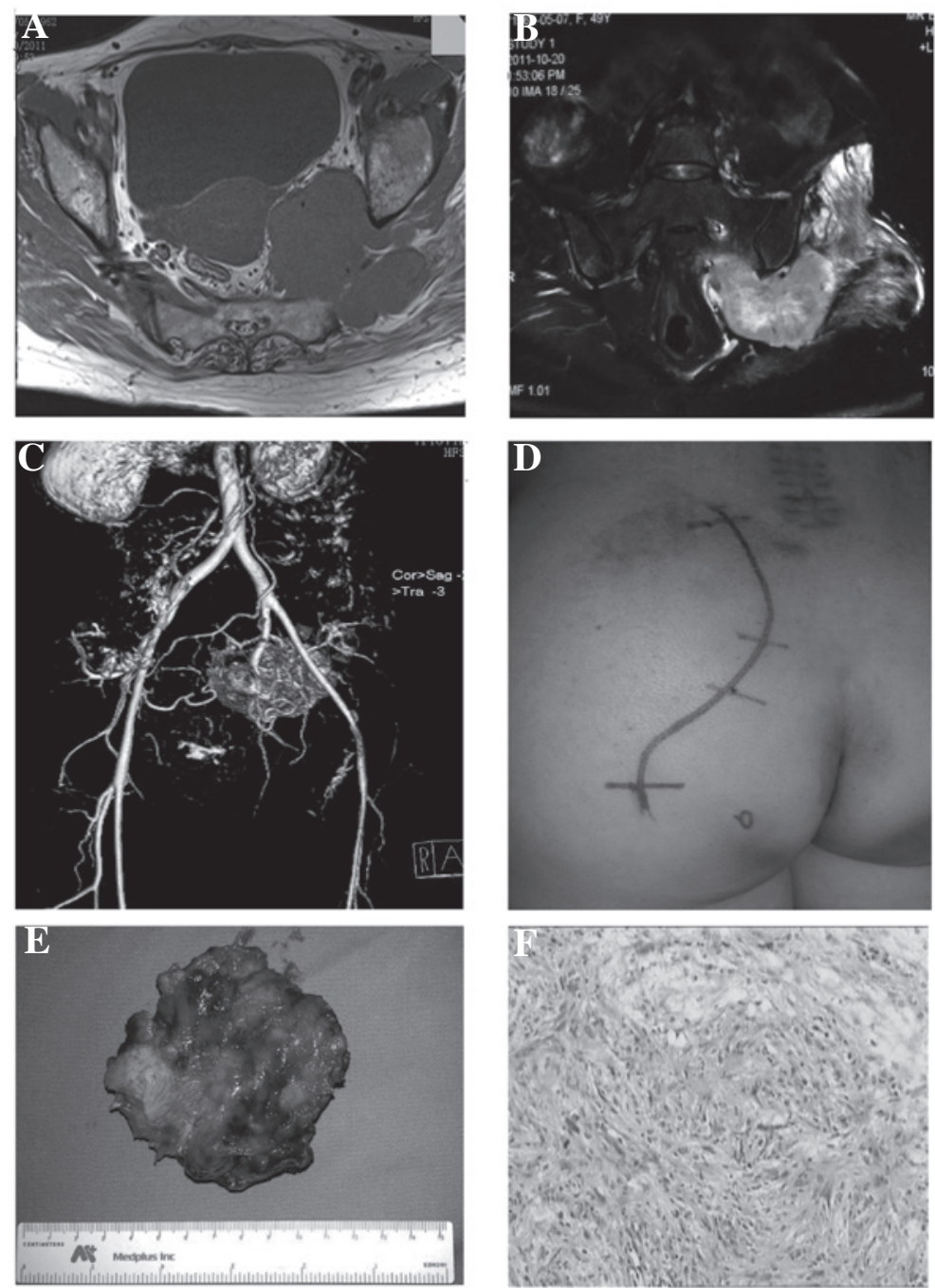

Figure 2. (A) T1-weighted axial pelvic magnetic resonance imaging (MRI) revealed a dumbbell-shaped isodense mass. The greater part of the mass was intrapelvic and the remainder was extrapelvic. (B) T2-weighted axial pelvic MRI revealed a dumbbell-shaped mass overpassing the sciatic notch. (C) Magnetic resonance angiogram of the pelvis revealed a $7 \mathrm{x} 8 \mathrm{-cm}$ mass involving the internal iliac artery. (D) Length of skin incision planned to expose the tumor. (E) Macroscopic appearance of the mass following surgical excision. (F) Hematoxylin and eosin staining of the tumor tissue (magnification, x100) showing a highly cellular tumor, displaying a sinuous pattern, with the tumor cells invading adipose tissue.

margins. When pelvic tumors extend outside the pelvis through the sciatic notch, a combined surgical approach, such as transabdominal plus gluteal approach, should be considered. The majority of benign pelvic tumors may be bluntly dissected after incising the epineurium over the mass. Unfortunately, for MPNSTs that involve the sciatic nerve or pelvic plexus, it is difficult to achieve complete removal of the mass with tumor-free margins without damaging the adjacent nerves. Wide local resection for MPNST including the sciatic nerve or pelvic plexus is required (13), as reported in our patient. Multimodality treatments, such as adjunctive radiotherapy and chemotherapy, have been associated with improved survival outcomes compared with previous reports $(14,15)$. However, only complete surgical excision prior to metastasis is likely to result in a good prognosis.

Patients with MPNST have a poor prognosis. Adverse prognostic factors include large size, high-grade tumor, proximal location, surgical margin with tumor invasion and NF1 $(16,17)$. The 5-year survival rates are associated with NF1. The reported 5-year survival rate for patients without NF1 may be as high as $50 \%$, whereas they may as low as $10 \%$ in patients with NF1 (18). Systemic spread occurs most commonly to the lungs, and pulmonary metastasis is the ultimate cause of death in cases of MPNST.

In summary, we herein report a rare case of giant intrapelvic MPNST misdiagnosed as intervertebral disc herniation. Our goal was to emphasize that intrapelvic MPNST, although infrequent, should be included in the differential diagnosis of sciatica, as its symptomatology may mimic disc herniation. Clinicians should consider the possibility of pelvic MPNST when patients present with obscure sciatica. The standard treatment of MPNST diagnosed early includes open biopsy, surgical treatment and a multidisciplinary approach to improve patient prognosis.

\section{References}

1. Topsakal C, Akdemir I, Tiftikci M, Ozercan I and Aydin Y: Malignant schwannoma of the sciatic nerve originating in a spinal plexiform neurofibroma associated with neurofibromatosis type 1-case report. Neurol Med Chir (Tokyo) 41: 551-555, 2001 
2. Kelso TB, Ferrari CJ and Frassica FJ: Sciatica caused by a neurilemoma of the intrapelvic portion of the sciatic nerve. A case report. J Bone Joint Surg Am 75: 603-605, 1993.

3. Naraghi MA: Neurofibroma of the sciatic nerve with neurofibromatosis type 1. Acta Med Iran 49: 480-482, 2011.

4. Zhu B, Liu X, Yang S, Liao H, Jiang L and Wei F: Malignant peripheral nerve sheath tumours of the spine: Clinical manifestations, classification, treatment, and prognostic factors. Eur Spine J 21: 897-904, 2012.

5. Wanebo JE, Malik JM, VandenBerg SR, Wanebo HJ, Driesen N and Persing JA: Malignant peripheral nerve sheath tumors. A clinicopathologic study of 28 cases. Cancer 71: 1247-1253, 1993.

6. Scheithauer BW, Erdogan S, Rodriguez FJ, Burger PC, Woodruff JM, Kros JM, Gokden M and Spinner RJ: Malignant peripheral nerve sheath tumors of cranial nerves and intracranial contents: A clinicopathologic study of 17 cases. Am J Surg Pathol 33: 325-338, 2009.

7. Amin A, Saifuddin A, Flanagan A, Patterson D and Lehovsky J: Radiotherapy-induced malignant peripheral nerve sheath tumor of the cauda equine. Spine (Phila Pa 1976) 29: E506-E509, 2004.

8. Kralick F and Koenigsberg R: Sciatica in a patient with unusual peripheral nerve sheath tumors. Surg Neurol 66: 634-637, 2006.

9. Rekha A and Ravi A: Sciatic nerve schwannoma. Int J Low Extrem Wounds 3: 165-167, 2004.

10. Richards JL and Matolo NM: Malignant schwannoma: Report of a case mimicking lumbar disk disease. Am Surg 45: 49-51, 1979.
11. White W, Shiu MH, Rosenblum MK, Erlandson RA and Woodruff JM: Cellular schwannoma. A clinicopathologic study of 57 patients and 58 tumors. Cancer 66: 1266-1275. 1990.

12. Kar M, Deo SV, Shukla NK, Malik A, DattaGupta S, Mohanti BK and Thulkar S: Malignant peripheral nerve sheath tumors (MPNST)-clinicopathological study and treatment outcome of twenty-four cases. World J Surg Oncol 4: 55, 2006.

13. Nawabi DH and Sinisi M: Schwannoma of the posterior tibial nerve: The problem of delay in diagnosis. J Bone Joint Surg Br 89: 814-816, 2007.

14. Baehring JM, Betensky RA and Batchelor TT: Malignant peripheral nerve sheath tumor: The clinical spectrum and outcome of treatment. Neurology 61: 696-698, 2003.

15. Stucky CC, Johnson KN, Gray RJ, Pockaj BA, Ocal IT, Rose PS and Wasif N: Malignant peripheral nerve sheath tumors (MPNST): The Mayo Clinic experience. Ann Surg Oncol 19: 878-885, 2012.

16. Angelov L, Davis A, O'Sullivan B, Bell R and Guha A: Neurogenic sarcomas: Experience at the University of Toronto. Neurosurgery 43: 56-65, 1998.

17. Anghileri M, Miceli R, Fiore M, Mariani L, Ferrari A, Mussi C, Lozza L, Collini P, Olmi P, Casali PG, et al: Malignant peripheral nerve sheath tumors: Prognostic factors and survival in a series of patients treated at a single institution. Cancer 107: 1065-1074, 2006.

18. Doorn PF, Molenaar WM, Buter J and Hoekstra HJ: Malignant peripheral nerve sheath tumors in patients with and without neurofibromatosis. Eur J Surg Oncol 21: 78-82, 1995. 